\title{
Análisis florístico en un gradiente altitudinal ubicado en el cerro Mecana, en el Chocó Biogeográfico
}

\section{Floristic analysis in an altitudinal gradient located in the Cerro Mecana, in the Chocó Biogeográfico}

\section{Reimer Rengifo-lbargüen*}

\section{Resumen}

El análisis de la vegetación en un gradiente altitudinal entre 200 y 650 msnm en el cerro Mecana, permitió registrar 488 individuos, pertenecientes a 38 familias, 88 géneros y 122 especies. Arecaceae, Melastomataceae, Sapotaceae, Clusiaceae, y Rubiaceae fueron las familias más abundantes en todo el gradiente con 79, 58, 37, 35 y 29 individuos respectivamente. La composición de especies y géneros disminuyó a medida que se asciende en el gradiente, mientras el número de familias fue poco variable. Esto como consecuencia de las características y particularidades expresadas a lo largo y ancho del ecosistema. Las estaciones E1 y E2, tienen similitud en un 75\% de las especies, y estas a su vez presentan $51 \%$ y $36 \%$ de similitud con la E4 y E3 respectivamente. La exclusividad de especies aumentó desde la estación más baja E1 a la E3 la cual registró 42\% de especies. Los hábitos de crecimiento muestran que $30 \%$ de los individuos son arbustos, 27\% árboles, 22\% hierbas y $21 \%$ palmas. Por lo tanto, la mayor cantidad de individuos se ubicó en las clases diamétricas inferiores mostrando comportamiento de " $J$ " invertida, es decir, muchos individuos juveniles y pocos árboles maduros. Las especies más representativas de acuerdo con el índice de valor de importancia (IVI) en el gradiente altitudinal evaluado fueron: Pouteria cuspidata con 24\% del IVI; Vismia macrophylla 13,9\%; Chrysochlamys floribunda 12,2\%; y Carapa guianensis 10,8\%; y Licania chocoensis con 10,3\% del IVI total. Al menos 15 especies revisten algún grado de amenaza y están representadas en las familias: Arecaceae, Apocynaceae, Chrysobalanaceae, Cyatheaceae, Humiriaceae, Malvaceae, y Myristicaceae.

Palabras clave: Análisis florístico, Cerro Mecana, Composición, Chocó Biogeográfico, Gradiente altitudinal.
* Biólogo, Instituto de Investigaciones Ambientales del Pacífico (IIAP), Quibdó, Colombia. e-mail: rrengifo@,iiap.org.co 


\section{Bioetnia Volumen 14, 2017}

\section{Summary}

The analysis of the vegetation in an altitudinal gradient between 200 and 650 meters above sea level in the Mecana hill, allowed registering 488 individuals, belonging to 38 families, 88 genera and 122 species. Arecaceae, Melastomataceae, Sapotaceae, Clusiaceae, and Rubiaceae were the most abundant families in the entire gradient with 79, 58, 37, 35 and 29 individuals respectively. The composition of species and genera decreased as the amount of the gradient increased, while the number of families was little variable. This, as a consequence of the characteristics and particularities expressed throughout the ecosystem. The stations E1 and E2, have similarity in 75\% of the species, and these in turn have 51 and $36 \%$ similarity with the E4 and E3 respectively. The exclusivity of species increased from the lowest station E1 to E3, which registered $42 \%$ of species. Growth habits show that $30 \%$ of individuals are shrubs, 27\% trees, 22\% herbs, and $21 \%$ palms. Therefore, the largest number of individuals, was located in the lower diameter classes showing inverted " $J$ " behavior, that is, many juvenile individuals and few mature trees. The most representative species according to the value index of importance (VII) in the altitudinal gradient evaluated were: Pouteria cuspidata with 24\% of the VII; Vismia macrophylla with 13.9\%; Chrysochlamys floribunda 12.2\%; and Carapa guianensis 10.8\%; and Licania chocoensis with 10.3\% of the total VII. At least 15 species have some degree of threat, and are represented in the families: Arecaceae, Apocynaceae, Chrysobalanaceae, Cyatheaceae, Humiriaceae, Malvaceae, and Myristicaceae.

Keywords: Altitudinal gradient, Cerro Mecana, Composition, Chocó

Biogeographic, Floristic analysis.

\section{Introducción}

La relación entre la riqueza de especies y la elevación, es un patrón ecológico que ha venido siendo documentado con frecuencia (MacArthur 1969, Colwell y Hurtt 1994, Schoener 1976, Stevens 1989, Kitayama 1992, Kitayama y Mueller-Dombois 1994, Kappelle y Zamora 1995, Rahbek 1995, Boyle 1996, Lieberman et al. 1996, Vázquez y Givinish 1998, Kessler 2000, Kessler et al. 2001, Koleff y Gaston 2001, Kessler 2002a, 2002a, Krömer et al. 2005, Cardelús et al. 2006, Kessler et al. 2011, Sanders y Rahbek 2012). Al respecto, Charles Darwin, Wallace y von Humboldt proporcionaron las primeras observaciones detalladas de cómo el mundo natural cambia con el gradiente altitudinal (Lomolino 2001). En sus viajes por el mundo, estos naturalistas anotaron que los tipos de hábitats y el número de especies cambiaban de manera impredecible con el aumento de la elevación y latitud (McCain y Grytnes 2010). De manera similar, otros autores manifiestan que la riqueza de especies decrece con el incremento de la elevación (MacArthur 1972, Stevens 1992, Richards 1996 citado por Leiva 2001), y otros afirman que existe un pico de riqueza en elevaciones intermedias (Stevens 1989, Rahbek 1995). Ambos patrones han sido documentados en una variedad de taxones y hábitats (Stevens 1992, Brown 1995, Rahbek 1995, Rosenzweig 1995, Brown y Lomolino 1998).

Todo lo anterior, deja ver como el comportamiento de la riqueza y composición de especies sobre el gradiente altitudinal, puede ser muy variable, pudiéndose encontrar tanto picos de riqueza en alturas intermedias, como disminución de esta riqueza a medida que se asciende en el gradiente. De acuerdo con Mendoza-Cifuentes (2012), la mayor parte de los trabajos en gradientes altitudinales se han concentrado hacia las zonas templadas y se han enfocado a un solo grupo de organismos. 
Bajo la perspectiva anterior y considerando que uno de los tópicos centrales en la ecología de comunidades es analizar la diversidad y composición florística en relación con el gradiente de elevación (Dobzhansky 1950, Ricklefs 2003, Mittelbach et al., 2007), en este artículo, se analiza como varía la vegetación en relación con un gradiente altitudinal en el cerro Mecana, a partir de la composición y estructura de la flora allí asociada. Mecana, es un cerro ubicado en el municipio de Bahía Solano, en las estribaciones de la Serranía del Baudó en el departamento del Chocó, el cual representa un ecosistema de media montaña, donde factores físicos como la humedad relativa, precipitación, temperatura y topografía juegan un papel fundamental en el establecimiento y mantenimiento de cada uno de los elementos bióticos que allí convergen (IIAP2014). Las dinámicas de ocupación y usos del territorio por parte de las diferentes etnias asentadas en el Chocó y en inmediaciones de sus cordilleras muestran la vulnerabilidad de los ecosistemas allí presentes, por lo que es necesario intentar comprender las dinámicas naturales de los elementos bióticos, en especial de la flora que resulta ser uno de los compartimentos más conspicuo del bosque que mejor refleja la afectación antrópica.

Para el cerro Mecana, son pocos los trabajos que en términos florísticos se han realizado, por lo que los datos documentados para esta zona se reducen prácticamente a los levantados por el Instituto de Investigaciones Ambientales del Pacífico en caracterizaciones biológicas (IIAP 2014) y monitoreos de la vegetación (IIAP 2016) que dan cuenta de la exuberante vegetación, composición y estado de conservación de algunas especies. Los pocos estudios en este cerro se ven en parte justificados, por la necesidad de conocer e investigar otros cerros igualmente importantes en la región chocoana. Tal es el caso de los cerros Jánano, Careperro, Chageradó, Mecana, Mutis, Alto del Buey, Galápagos y Tacarcuna (este último cerro fue recientemente explorado en el marco de las expediciones Colombia Bio). Estos estudios (IIAP 2011, 2013, 2014, 2015), a pesar de no analizar la relación flora-gradiente altitudinal, sí escudriñan sobre la composición, estructura vegetal y en algunos casos sobre especies de interés especial; no obstante, en la caracterización del Alto del Buey (IIAP y CODECHOCÓ 2011) es posible entender, que a medida que se asciende en el gradiente altitudinal, la diversidad de especies disminuye o se ve ligeramente mezclada con especies típicas de áreas de mayor altitud.

Así entonces, los trabajos realizados en este sitio(IIAP 2012) y suárea de influencia (Yockteng y Cavelier 1998, Mosquera et al. 2007), aunque se han focalizado a determinar de alguna manera su composición florística, no analizan como varían estos elementos florísticos con respeto al gradiente altitudinal. Por tanto, en este artículo se investiga básicamente cómo varía la vegetación en términos de composición y estructura respecto al gradiente altitudinal en el cerro Mecana. Además, se hacen algunas anotaciones sobre la flora de interés especial registrada, como estrategia para dimensionar aún más su importancia y generar alertas tempranas sobre los posibles impactos de las acciones antrópicas en este importante ecosistema.

\section{Materiales y métodos}

Area de estudio. El cerro Mecana se encuentra localizado en el municipio de Bahía Solano, en la costa norte del departamento del Chocó, a los $6^{\circ} 15^{\prime} 53.91$ 'N, y $77^{\circ} 18^{\prime} 9.21^{\prime \prime} \mathrm{W}$, sobre la serranía del Baudó. Según el sistema de Holdridge (1986), esta zona corresponde a bosque muy húmedo Tropical (bmh-T) y bosque pluvial Tropical (bpT). Sus suelos son derivados de rocas basálticas y consideradas moderadamente desarrolladas, ácidos y fuertemente lixiviados (Cortés 1981, 1993). El promedio de temperatura anual es de $26^{\circ} \mathrm{C}$ y su precipitación media anual de $5.100 \mathrm{~mm}$.

Mecana, constituye un ecosistema de media 


\section{Bioetnia Volumen 14, 2017}

montaña, con unos 1.150 metros de altura, en el cual tienen cabida una cantidad de procesos ecológicos y coexisten una diversidad de especies tanto de flora como de fauna (IIAP 2014). Este ha sido un territorio donde por siglos, diferentes comunidades indígenas y negras, han permanecido en un aislamiento natural y cultural con el interior del país, lo que ha permitido que sus tradiciones y relación con el medio ambiente conserven muchas de sus características ancestrales, dando lugar en gran medida a la conservación y presencia de la diversidad biológica y paisajística actual. Hace más de 9 años este cerro fue objeto de extracción selectiva de madera para actividades de subsistencia de estas comunidades.

Muestreo. Para caracterizar la vegetación asociada con el cerro Mecana, se delimitó un gradiente altitudinal comprendido entre los 200 y $750 \mathrm{msnm}$. A partir de este gradiente, se establecieron cuatro estaciones de muestreo (E1, E2, E3, E4), ubicadas a una distancia aproximada de $150 \mathrm{~m}$ altitudinales, iniciando desde los 200 msnm en la estación más baja. En cada unidad de muestreo, se instalaron 4 parcelas de $4 \mathrm{~m} \mathrm{x}$ $25 \mathrm{~m}\left(100 \mathrm{~m}^{2}\right)$, ubicadas estratégicamente a una distancia aproximada de $30 \mathrm{~m}$ una de la otra y divididas cada una en cinco subparcelas de $4 \mathrm{x}$ $5 \mathrm{~m}$. Las subparcelas fueron referenciadas con números del 1 al 5 . La vegetación arbustiva se censó en las subparcelas 1, 3 y 5, mientras la vegetación herbácea en la 2 y la 4 .

Las estaciones de muestreo presentaron las siguientes características: La E1, se ubicó hacia la parte baja del cerro sobre los $200 \mathrm{msnm}$, donde se alcanzan a diferenciar tres estratos de bosque y predominan diferentes árboles con un dosel semicerrado de especies maderables como $\mathrm{Hu}$ berodendron painoi Cuatrec., Carapa guianensis Aubl., y Anacardium excelsum (Bertero \& Balb. ex Kunth) Skeels, en asocio de una variedad de palmas como Wettinia quinaria (O.F. Cook \& Doyle) Burret., Welfia regia Mast., Astrocaryum standleyanum L.H. Bailey entre otras. El soto- bosque lo integran junto con algunas palmas, especies del género Matisia (Malvaceae). En el estrato herbáceo, predominaron hierbas bastante conspicuas de la familia Marantaceae. El suelo posee abundante materia orgánica y evidencia procesos de extracción forestal de hace algunos años.

La E2, se ubica por encima de los $350 \mathrm{msnm}$, integra un ambiente variado en cuanto a hábitos de crecimiento, con la presencia de palmas del género Geonoma y especies forestales como Calophyllum mariae Planch. \& Triana y Aspidosperma cruentum Woodson. Varias especies de los géneros Miconia, Psychotria y algunas especies de Araceae, así como epífitas de la familia Gesneriaceae. Tiene poca incidencia de rayos solares, la geomorfología es irregular con pendientes hasta de $35^{\circ}$ de inclinación, y abundante materia orgánica.

La E3, se ubica por encima de los $500 \mathrm{msnm}$, y se ve representada por especies como Aspidosperma cruentum, y Licania platypus (Hemsl.) Fritsch, combinadas con Psychotria elata, Psychotria cinta, Psychotria cf. marginata y Protium nervosum Cuatrec., seguida de vegetación arbustiva y helechos de la familia Tectariaceae. Se observan algunos claros de bosque al parecer originados por árboles caídos y otros muertos en pie. La topografía es irregular y el suelo presenta pendientes hasta de $50^{\circ}$ de inclinación, posee abundante materia orgánica.

Por último, la E4, se ubica sobre los $650 \mathrm{msnm}$, y exhibe especies como Licania sp., que alberga y sirven de soporte a algunas especies de epífitas (bromelias y Gesneriaceae) y briófitos los cuales aumentan su presencia en árboles y arbustos; aparece en mayor cantidad individuos de la especie Voyria tenella (Gentianaceae) indicadora de sitios en buen estado de conservación. Algunos claros de bosque permiten el surgimiento de especies de palmas y Melastomataceae. La topografía muestra pendientes de hasta $45^{\circ}$ sosteniendo árboles con más de $30 \mathrm{~m}$ de altura. La vegetación herbácea 
se reduce a especies de los géneros Faramea y Psychotria, acompañadas de muchos individuos del género Miconia.

En cada parcela, se tomaron los siguientes datos para cada individuo con un diámetro basal $\geq 2 \mathrm{~cm}$ a $30 \mathrm{~cm}$ del suelo: altura total del individuo, altura a la primera ramificación (árboles), y forma de crecimiento(árbol, arbusto, hierba, epífita, palma). Luego se colectaron entre dos y tres ejemplares de cada morfoespecie, preferiblemente en estado fértil, los cuales fueron preparados de acuerdo con los métodos estandarizados de material vegetal para un herbario, para posteriormente ser depositados en el Herbario CHOCÓ de la Universidad Tecnológica del Chocó en Quibdó.

Procesamiento y análisis de la información. La identificación del material colectado se realizó con base en las colecciones del Herbario CHOCÓ, literatura taxonómica como la de Gentry (1993), Mendoza y Ramírez (2006), Mendoza et al. (2004), y las bases de datos de: Herbario Nacional Colombiano (COL), Jardín Botánico de Missouri (MO), New York Botanicals Garden (NY), Real Jardín Botánico (KEW); así como International Plant Names Index (IPNI), Neotropical Herbarium Specimens http://fm.fieldmuseum.org/vrrc. Los nombres de los taxones obtenidos fueron ingresados, validados y depurados en TNR (Taxonomic Name Resolution Service) (Boyle et al. 2013) y en www.tropicos.org.

Para estimar la diversidad florística del gradiente, a cada estación de muestreo se le calculó el índice de diversidad de Shannon-Wiener, dominancia de Simpson y la riqueza de especies (definida como el número de especies). La estructura de la vegetación se analizó a partir de la distribución del número de árboles por clase diamétrica en cada estación, así como mediante el cálculo de la densidad total, frecuencia relativa, áreas basales, e IVI; para la caracterización vertical de la vegetación se siguió la propuesta de Rangel y Lozano (1986), citada en Villareal et al. (2006), que propone los siguientes estratos según su altura total: rasante $(<0,3 \mathrm{~m})$, herbáceo (0,3-1,5 m), arbustivo (1,5-5 m), subarbóreo o de arbolitos (5-12 m), arbóreo inferior (12-25 m) y arbóreo superior $(>25 \mathrm{~m})$. El recambio de especies en el gradiente se realizó mediante un análisis de agrupamiento clúster, a través del índice de Jaccard y Bray-Curtis. Todo esto con la ayuda del programa PAST.

Para la tipificación de la abundancia o densidad de las especies presentes en el gradiente, se utilizó la clasificación propuesta por Oliveira y Rotta (1982) por la cual, especies representadas entre 1 y 6 individuos corresponden a raras o escasas, aquellas entre 7 y 25 se consideran poco abundantes y especies con más de 25 individuos se clasifican como abundantes.

Por otra parte, se analizó el estado actual de la vegetación y se determinaron las especies de interés especial, con base en la información obtenida en las parcelas, muestreos al azar, y datos de la caracterización realizada en el año 2014 (IIAP 2014).

\section{Resultados y discusión}

Composición florística en el gradiente altitudinal. Se registraron en total 488 individuos para el gradiente altitudinal, pertenecientes a 38 familias, 88 géneros y 122 especies (Anexo). Estos datos, resultan muy diferentes, si se comparan con los descritos para cerros aledaños. Tal es el caso del cerro Murrucucú, donde el IIAP (2014) reporta 21 familias, 35 géneros y 36 especies. De igual forma, para el cerro Mutis, se reporta 34 familias, 74 géneros y 88 especies (IIAP 2015). Tales diferencias, aunque pueden estar influidas por el tamaño del área inventariada, muestran la buena representatividad del cerro Mecana como parte de la serranía del Baudó. Por otra parte, la riqueza de este ecosistema dista poco de lo reportado por otros autores para áreas de influencia del cerro. Por ejemplo, Mosquera et al. (2007) registraron en dos localidades del municipio Alto 


\section{Bioetnia Volumen 14, 2017}

Baudó ubicadas a menos altura sobre el nivel del mar, 45 familias, 98 géneros y 161 especies en el corregimiento Nauca, y 46 familias, 108 géneros 161 especies en Pie de Pató; el IIAP y CODECHOCÓ (2011), reportaron 153 especies representadas en 109 géneros y 58 familias para el Alto del Buey y sus inmediaciones; y para el cerro Jánano, el IIAP(2013) reportó 161 especies agrupadas en 123 géneros y 42 familias.

Respecto a la composición de especies en el gradiente, la estación E1, estuvo representada por 143 individuos distribuidos en 30 familias, 65 géneros y 73 especies, donde las familias más abundantes por número de individuos fueron en su orden: Arecaceae (35), Melastomataceae (17), Sapotaceae (15), seguidas de Moraceae y Clusiaceae con 7 cada una. Las familias mejor representadas en cuanto a número de especies fueron: Arecaceae (18), Melastomataceae con 5, seguidas de Apocynaceae, Rubiaceae y Moraceae con 4 cada una; estas familias agruparon $44 \%$ de las especies presentes en esta estación. A nivel de especies, se destacan por su abundancia Pouteria cuspidata (12 individuos), Miconia nervosa (11), Wettinia quinaria, Carapa guianensis y Chrysochlamys floribunda con 5 individuos cada una; estas especies suman en total $26 \%$ de los individuos.

LaE2 por su parte, registró 117 individuos distribuidos en 26 familias, 56 géneros y 61 especies. Las familias con mayor cantidad de individuos fueron en su orden: Arecaceae (35), Hypericaceae (11), y Rubiaceae (9). Las familias mejor representadas en cuanto a número de especies fueron Arecaceae (19), Rubiaceae (8), Apocynaceae, Clusiaceae, Lauraceae, y Malvaceae con 4 cada una. Estas familias agruparon $62 \%$ de las especies presentes en esta estación. A nivel de especies, se destacan por su abundancia Wettinia quinaria con 13 individuos, Vismia macrophylla (11), Andira surinamensis (8), y Asterogyne martiana y Oenocarpus bataua con 5 individuos cada una; estas especies suman en total $36 \%$ de los individuos.
La E3 estuvo representada por 138 individuos distribuidos en 27 familias, 42 géneros y 52 especies. Las familias con mayor cantidad de individuos fueron en su orden: Melastomataceae (34), Clusiaceae (21), Sapotaceae con 15 y Tectariaceae con 11. Las familias mejor representadas en cuanto a número de especies fueron Arecaceae con 8 , Araceae y Sapotaceae con 5 cada una, seguida de Melastomataceae con 4 en cada caso. Estas familias agruparon 50\% de las especies presentes en esta estación. A nivel de especies, se destacan por su abundancia Clidemia aff hirta con 29 individuos, Chrysochlamys floribunda con 21, seguidas de Pouteria cuspidata y Tectaria sp con 11 individuos cada una; estas especies suman cerca de $70 \%$ del total de individuos en la estación.

La E4 estuvo representada por 90 individuos distribuidos en 23 familias, 30 géneros y 38 especies. Las familias con mayor cantidad de individuos fueron en su orden: Rubiaceae (11), Lecythidaceae (8), seguidas de Melastomataceae, Myristicaceae y Apocynaceae con 7 cada una. En cuanto a número de especies fueron las más diversas Fabaceae y Rubiaceae con 3, seguida de Araceae, Chrysobalanaceae, y Clusiaceae con 2 en cada caso. Estas familias agruparon $29 \%$ de las especies presentes en esta estación. A nivel de especies, se destacan por su abundancia Miconia nervosa y Aspidosperma desmanthum con 6 cada una; Carapa guianensis, Protium nervosum, y Tectaria sp. con 5 individuos cada una para un total del 30\% de los individuos.

A nivel de todo el gradiente altitudinal, Arecaceae fue la familia con mayor número de individuos (79), seguida de Melastomataceae con 58, Sapotaceae con 37, Clusiaceae con 35 y Rubiaceae con 29. Las familias con mayor número de especies fueron en su orden: Arecaceae (19), Rubiaceae (10), Araceae (8), Sapotaceae (7) y Melastomataceae y Myristicaceae con 6 cada una. Aunque este patrón de familias no se mantuvo en las diferentes estaciones, la familia Arecaceae marcó la diferencia porque, en términos 


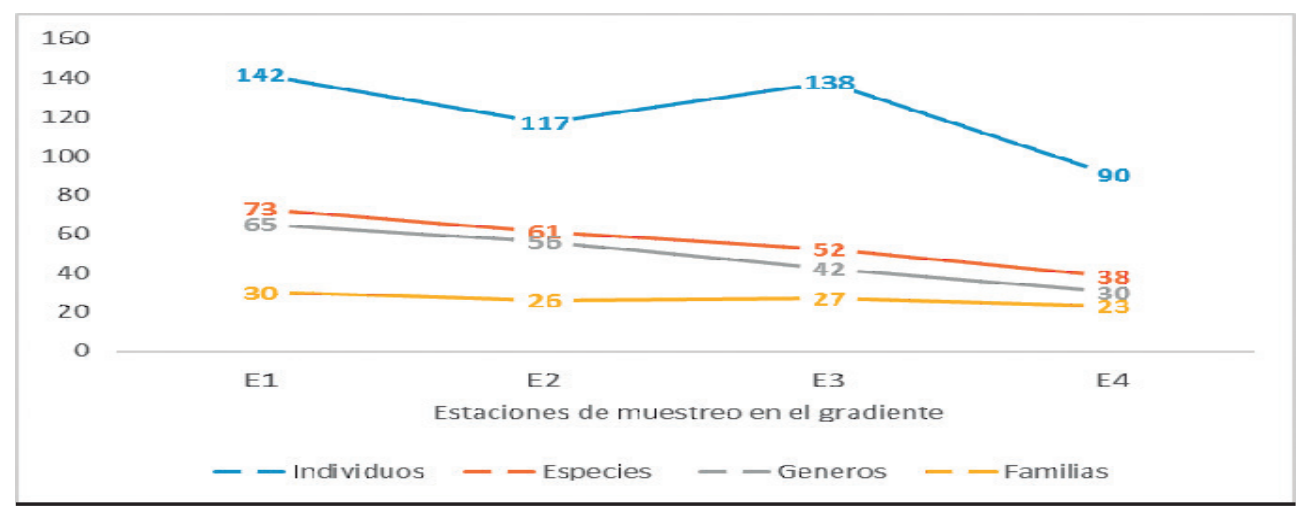

de especies, mantuvo su representatividad en todo el gradiente altitudinal.

La tipificación de la abundancia en el gradiente, de acuerdo con la clasificación de Oliveira y Rotta (1982), arrojó que 112 especies son escasas, 9 son poco abundantes, y solo 3 especies (Clidemia aff hirta, Chrysochlamys floribunda y Pouteria cuspidata) resultaron abundantes por haber registrado más de 25 individuos. A pesar de esto, solo Pouteria cuspidata se distribuyó en todas las estaciones.

La diferencia más marcada entre las cuatro estaciones tiene que ver con el número de individuos; no obstante, estas diferencias no parecen obedecer al gradiente altitudinal, sino al efecto de los árboles recientemente caídos registrados en la E2 y E4 que limitaron las posibilidades de censar más individuos; esta situación puede verse compensada en el tiempo, debido a las plántulas que pueden emerger de manera natural en los claros originados por estos árboles caídos. Sin embargo, la riqueza de especie y géneros, si se relacionan con la variación del gradiente, mostrando que a medida que se asciende en el cerro, el número de estos taxones disminuye. Este comportamiento ratifica parcialmente lo manifestado por autores como Bertin et al. (2003), Villar y Benito (2003), y Erschbamer et al. (2006), citados por IIAP (2014), quienes argumentan que el número de taxa pueden aumentar o disminuir en función del gradiente altitudinal. El número de familias fue mayor en las estaciones E1 y E3, sin embargo, no hubo diferencias marcadas en relación con las demás estaciones (Figura 1 ).

La disminución gradual de las especies y géneros, con el aumento de altitud en el gradiente, permite estimar que en este cerro y bajo las condiciones actuales del hábitat y evaluado con metodologías similares, las especies pueden llegar a verse reducidas gradualmente hasta en un $20 \%$ cada $300 \mathrm{~m}$ altitudinales, hasta llegar a la cima del gradiente. Esto, como consecuencia de las características y particularidades expresadas a lo largo del ecosistema. Sin embargo, de acuerdo con lo manifestado por Stevens (1989) y Rahbek (1995), la variación de especies y géneros puede mostrar picos de riqueza en elevaciones intermedias.

Con base en lo anterior, y de acuerdo con el análisis de agrupamiento clúster (Figura 2), se puede apreciar un grupo principal, formado por las E1 y E2, que tienen una similitud en el 75\% de las especies; mientras tanto, las E4 y E3 son más disímiles con respecto a este grupo, pues solo presentan entre $51 \%$ y $36 \%$ de especies compartidas respectivamente. Tal situación informa no solo sobre picos de riquezas en altura intermedias, sino sobre la diferenciación específica, a medida que se asciende en el gradiente.

En relación con las familias más abundantes por número de individuos en el gradiente altitudinal (Arecaceae 79, Melastomataceae 58, Sapotaceae 37, Clusiaceae 35 y Rubiaceae con 


\section{Bioetnia Volumen 14, 2017}

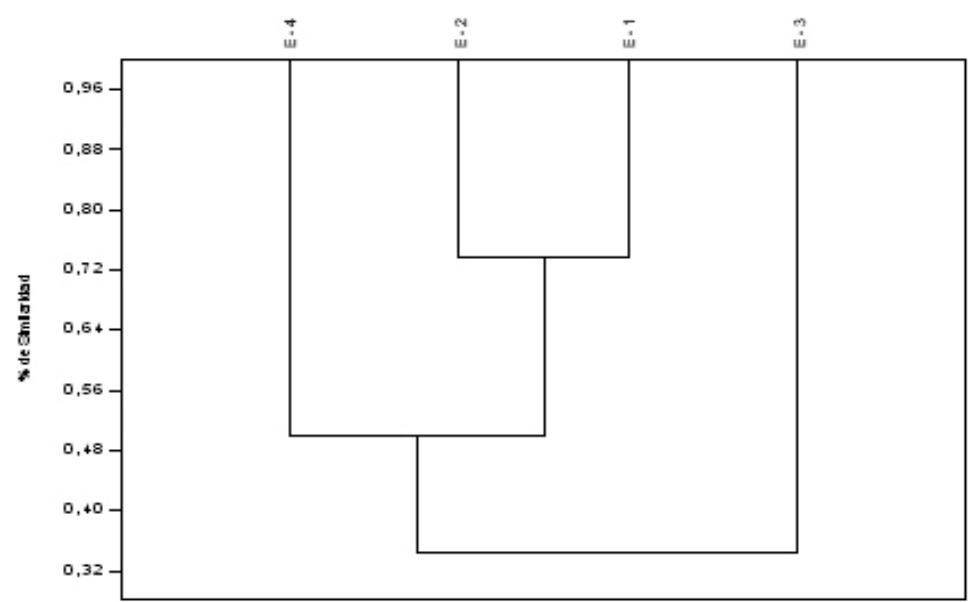

Figura 2. Análisis de agrupamiento de las estaciones de muestreo, de acuerdo con el número y cantidad de especies registradas. Nótese la mayor similitud entre la E1 y la E2.

29), se estableció que todas tienen presencia en el gradiente muestreado; no obstante, hay una diferenciación en las abundancias en cada una de las estaciones. Por ejemplo, para el caso de las familias Arecaceae y Melastomataceae, se halló que sus individuos, tienen mayor presencia en las E3 y E4, pero los pocos individuos de las E1 y E2, representan mayor diversidad de especies (10 en Arecaceae: Wettinia quinaria, Oenocarpus bataua, Socratea exorrhiza, Asterogyne martiana, Oenocarpus minor, Welfia regia, Bactris barronis, Ischnosiphon arouma, Welfiageorgii, Phytelephas seemannii; y 3 en Melastomataceae: Miconia nervosa, Miconia sp., Miconia punctata). Un estudio en otro rango altitudinal sobre la familia Melastomataceae (Mendoza-Cifuentes 2012), muestra que solo presenta una disminución significativa de la riqueza en localidades por encima de los $2800 \mathrm{~m}$ de altitud, es decir, en zonas de subpáramo y páramo.

De manera similar sucede con la familia Sapotaceae, que tiene menos individuos en las E1 y E2, pero es ahí donde presenta mayor número de especie (6: Pouteria cuspidata, Chrysophyllum argenteum, Aspidosperma desmanthum, Pouteria multiflora, Chrysophyllum cainito; e igualmente con Clusiaceae, que a pesar de los 23 individuos registrados en las estaciones E3 y E4, solo representaron una especie (Crysophyllum oliviforme).
Para el caso de Rubiaceae, esta familia resultó ser la única entre las más abundantes, que mostró equidad entre las estaciones.

Lo anterior se puede relacionar con la deposición de nutrientes, debido a la lixiviación desde las partes altas del cerro, que pueden contribuir a la diversificación en las estaciones ubicadas a menor escala altitudinal. Esta situación, ayuda a entender por qué la diversidad de especies y géneros en el cerro Mecana disminuye con el incremento altitudinal.

Distribución de especies en el gradiente. Se estableció que del total de especies, 53 (48\%) se mostraron restringidas a una sola estación, 45 $(37 \%)$ a dos, $17(14 \%)$ a tres y solo $6(5 \%)$ especies se distribuyen a través de todo el gradiente muestreado. Lo anterior muestra que una buena parte de las especies tienen exclusividad o preferencia por un tipo de ambiente y que, por tanto, existen muy pocas que son generalista, al menos en la parte del gradiente monitoreado. Tal situación puede relacionarse con algunas variaciones existentes entre las estaciones como: topografía, nivel de pendiente, msnm, claros naturales de bosque, abundancia de materia orgánica e incidencia de rayos solares, lo que puede determinar las características del hábitat y el establecimiento de algunas especies.

Por otro lado, se apreció una ligera exclusivi- 


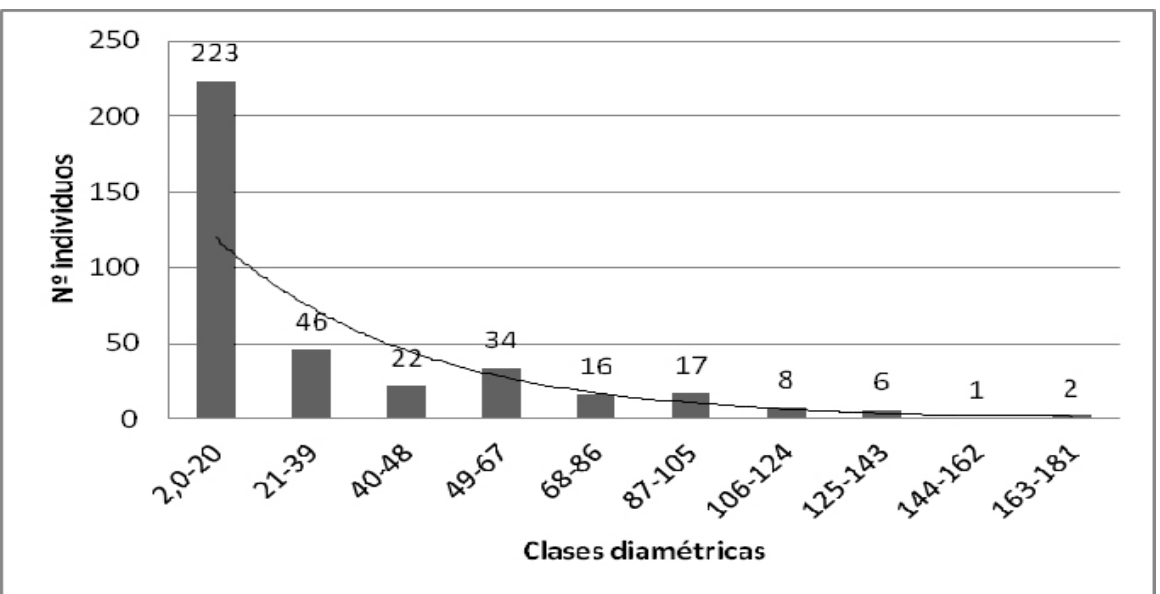

Figura 3. Distribución de los individuos por clase diamétrica. Nótese la menor abundancia en las clases diamétricas inferiores.

dad de especies en el gradiente altitudinal; para la E1, $9(16 \%)$ especies resultaron exclusivas (Otoba novogranatensis, Pholidostachys dactyloides, Psychotria marginata, Tessmanianthus calcaratus, Astrocaryum standleyanum, Bactris coloniata, Blakea podagrica, Garcinia madruno, Guzmania glomerata, Guzmania linguilata, Guzmania musaica, Inga vera); para la E2 15 (24\%) (Pentaclethra macroloba, Prestodea decurrens, Psychotriaelata, Psychotriapilosa, Rhodospatha moritziana, spidosperma cruentum, Tovomita weddelliana, Welfia georgii, Garcinia madruno, Dussia lechmally, Eschweilera pittieri, Geonoma deversa, Guatteria cargadero, Guettarda crispiflora subsp. Discolor, y Huberodendron patinoi); para la E3 22 (42\%) (Licania chocoensis, Licania platypus, Licaria triandra, Miconia puntata, Mouriri sp, Otoba lehmannii, Philodendron fenzlii, Pouteria cuspidata, Psychotria capitata, Syngonium sp., Syngonium triphyllum, Anthurium hacumense, Anthurium lancifolium, Ardisia sp., Bursera tomentosa, Chrysophyllum cainito, Clidemia aff hirta, Cordia sp., Euterpe sp., Geonoma cuneata, Geonoma estricta), y para la E4, $20 \mathrm{spp}$. que representan $40 \%$.

Lo anterior manifiesta no solo la importancia de cada zona por sus especies particulares, sino que denota las particularidades y características intrínsecas de cada una como parte del gradiente, las cuales son determinantes en el patrón de distribución de la diversidad. Por otra parte, a pesar de que la diversidad de especies se reduce al aumentar la altura sobre el nivel del mar, la exclusividad de estas especies tiende a aumentar. Así entonces, se muestra una relación proporcional entre el incremento altitudinal y la rareza de especies.

Estructura de la vegetación en el gradiente. Los 488 individuos registrados en el cerro representan un área basal de $11.181 \mathrm{~m}^{2}$; la mayor área basal la registró Pouteria cuspidata con 10,87 $\mathrm{m}^{2}$, seguida de Vismia macrophylla y Carapa guianensis con 6,83 y $6,42 \mathrm{~m}^{2}$ respectivamente. La distribución de los individuos por clases diamétricas mostró un comportamiento similar a una curva en forma de "J" invertida, ubicándose la mayor cantidad de individuos en las clases diamétricas inferiores, reflejando la presencia de muchos individuos jóvenes y pocos árboles maduros (Figura 3). La mayoría de individuos se distribuyen en la primera clase $(2-20 \mathrm{~cm})$. Esta distribución representa la tendencia del bosque a buscar la fase de homeostasis (equilibrio entre lo que se muere y lo que crece); lo que se entiende, por los movimientos que se presentan debido al paso de individuos entre las clases, debido a la dinámica natural del bosque, en donde la cantidad de individuos que logran establecerse durante los primeros años, va disminuyendo conforme 


\section{Bioetnia Volumen 14, 2017}

aumenta la clase diamétrica producto de la competencia intra e inter-específica y de las exigencias lumínicas que requieren algunas de las especies para obtener un sitio dentro el bosque (Rodríguez y Brenes 2009). Al respecto, Arruda et al. (2011) y Hernández-Stefanoni et al. (2011), manifiestan que la tendencia de este modelo de curva, también señala que la comunidad vegetal se encuentra en coherente proceso de desarrollo con dirección a etapas de crecimiento y productividad vegetal más avanzadas.

Por otra parte, estos resultados de acuerdo con lo planteado por Pardo y Cediel (1994), pueden ser producto de la interacción de factores como: suelos permanentemente lavados y bajo contenido de nutrientes que no permiten el sostenimiento de árboles de tamaño grande, y la topografía escarpada del terreno que influye sobre la dinámica del bosque y favorece la presencia de individuos con diámetros menores, lo que puede relacionarse también con los procesos de extracción selectiva de especies, evidenciados hacia la parte baja del gradiente.

Aunque la panorámica general del bosque muestra una buena presencia y representación de árboles sobre el gradiente, es de anotarse que al interior de las parcelas establecidas, se apreció muy pocos individuos con este hábito de crecimiento; el patrón irregular de distribución y la distancia promedio entre árboles $(2 \mathrm{~m}$ en promedio) dio cabida al registro de muy pocos individuos por cada parcela e inclusive por estación de muestreo. Por tanto, teniendo en cuenta el trabajo del IIAP (2014), se puede decir que el muestreo bajo la metodología de parcelas puede limitar el hallazgo de la cantidad real de árboles en el gradiente, porque el IIAP a pesar de haber muestreado un poco más arriba (hasta los 1.000 msnm) donde disminuyeron los árboles en este estudio, encontraron que los árboles fueron los más abundantes en todo el gradiente, sobre todo hacia la parte baja del mismo.

La vegetación registrada en el gradiente mos- tró diferentes formas de crecimiento (árboles, arbustos, hierbas y palmas), donde 30\% de los individuos son arbustos, $27 \%$ árboles, 22\% hierbas y $21 \%$ palmas. Estas formas de crecimiento se distribuyeron a lo largo de todo el gradiente altitudinal. No obstante, las cuatro estaciones mostraron algunas diferencias en relación con el porcentaje y patrón de hábitos que exhibe en su conjunto el área estudiada. Para el caso de los árboles, su mayor porcentaje (35\%) se registró hacia la parte baja (E1) y media (E2) del cerro, donde especies como Carapa guianensis, $\mathrm{Hu}$ berodendron patinoi, y Humiriastrum procerum fueron las más abundantes. La significativa presencia de árboles en esta parte del cerro, sumado a las presiones antrópicas por extracción forestal, muestran no solo la madurez del bosque y su estado de conservación, sino el potencial forestal del cerro y la necesidad de diseñar estrategias para su manejo, pues su cercanía a los centros poblados incrementa la vulnerabilidad de este ecosistema a las presiones antrópicas. Las hierbas por su parte abundaron más en la E3 (23\%) y E4 (28\%), mostrando a las especies de la familia Rubiaceae como las más abundantes; de manera similar, las palmas, mostraron la mayor concentración de individuos (40\%) en esa parte del gradiente.

Índice de valor de importancia de las especies. Aunque todas las especies son de suma importancia para mantener la dinámica del bosque en el cerro, tanto en estructura como en composición (Lamprecht 1990), las especies más representativas de acuerdo con el índice de valor de importancia en el gradiente altitudinal evaluado fueron: Pouteria cuspidata con $24 \%$ del IVI; Vismia macrophylla 13,9\%; Chrysochlamys floribunda 12,2\%; Carapa guianensis 10,8\%; y Licania chocoensis con 10,3\% del IVI total. E1 peso ecológico de $P$. cuspidata en el gradiente, estuvo influido por su frecuencia en las cuatro estaciones y en gran medida por la abundancia y área basal en la E4, donde registró un 60\% de su abundancia y $50 \%$ del área basal. Por tanto, estas 
especies resultan ser las que pueden estar adsorbiendo más nutrientes y controlando el mayor porcentaje de la energía que llega al ecosistema, lo que significa que su ausencia generaría cambios sustanciales en su estabilidad. Estos datos, se relacionan parcialmente con lo reportado por el IIAP (2014), quien también registró en el cerro Mecana, la dominancia de Pouteria cuspidata, Brosimum utile, y Humiriastrum procerum sobre las demás especies. Lo que pudo atribuirse a que son especies típicas de estas zonas, poseen un valor económico bajo y no son actualmente aprovechadas por los habitantes de las comunidades aledañas a estos bosques. El valor de importancia de las otras especies dependió principalmente de suárea basal. Vismia macrophylla, estuvo presente en tres estaciones; no obstante, cerca del $80 \%$ de su abundancia y $50 \%$ del área basal de esta especie se registró en la E2, mientras Chrysochlamys floribunda se registró en tres estaciones y 55\% del área basal lo aportó en la E4.

$\mathrm{Si}$ se analiza el peso ecológico por familias, Arecaceae con 39,2\% del IVI fue la más importante, seguida de Sapotaceae 38,5\%, Lauraceae $22,6 \%$, Myristicaceae 19,1\% y Chrysobalanaceae con $18,7 \%$. Otras familias con menor IVI, son en su orden: Apocynaceae con 15.1\%, Malvaceae $12.2 \%$, Melastomataceae $11.8 \%$, y Burseraceae $7.5 \%$. Estos datos coinciden parcialmente con los de Asprilla et al. (2003), quienes reportan a Arecaceae, Sapotaceae, Myristicaceae, Chrysobalanaceae, Burseraceae y Apocynaceae entre las familias más importantes a nivel ecológico en un bosque pluvial tropical en el municipio de Salero, Chocó, ubicado sobre los $75 \mathrm{msnm}$. Lo anterior deja ver que la importancia ecológica de estas familias, no se relaciona con el gradiente altitudinal, sino que puede obedecer a otros factores como:

Índices ecológicos. De acuerdo con el índice Shannon-Wiener, la mayor diversidad se presentó en la estación E1 $(3,962)$, seguida de la E2 $(3,71)$, E3 $(3,162)$, y E4 $(3,35)$. Lo que muestra que la diversidad disminuye a medida que se asciende sobre el gradiente altitudinal. Estos resultados coinciden con lo informado por Kessler et al. (2001), Nathan (2002), y Sanders y Rahbek (2012), quienes manifiestan que la riqueza específica decrece con el incremento de la altitud, y que es más común la presencia de un pico (hump-shaped) o franja intermedia con mayor riqueza, que un decrecimiento lineal y que no hay un solo mecanismo general que explique la variación altitudinal (Kessler et al. 2001, Nathan 2002, Sanders y Rahbek 2012).

En este sentido, Gaston et al. (1995) plantean que la región tropical es donde se concentra la mayor diversidad biológica del planeta, la cual decrece a medida que se avanza desde el Ecuador Geográfico hacia los polos. Por otra parte, estos resultados difieren con lo manifestado por Sanders y Rahbek (2012), quienes revisando más de 90 artículos encontraron el reporte de gradientes de diversidad en forma de joroba, con la mayor diversidad en alturas medias. No obstante, si se estima la riqueza considerando la máxima altura del cerro (cerca de $1.200 \mathrm{msnm}$ ) se encuentra que esta puede llegar a presentar un comportamiento análogo a lo manifestado por este autor.

La dominancia, basada en el índice de Simpson, osciló entre 0,97 y 0,96 presentando su mayor valor en la E1 y el menor en la E4. Respecto a la equidad, se puede notar que presentó valores que disminuyeron a medida que se incrementa la altura del gradiente, 0,9235 y 0,94 en las estaciones E1 y E4; en cambio, para las estaciones E2 y E3 fue menor, mostrando valores de 0,9028 y 0,8002 respectivamente (Tabla 1).

Flora de interés especial en el gradiente altitudinal. De acuerdo con la composición de especies registrada para este cerro, solo 15 revisten importancia o interés de conservación por encontrarse en alguna categoría según la Resolución 0192 (MADS 2014) o en el Apéndices de la CITES. Estas especies, se distribuyen en siete familias (Arecaceae, Apocynaceae, Chrysobalanaceae, Cyatheaceae, Humiriaceae, Malvaceae, 
Bioetnia Volumen 14, 2017

Tabla 1. Índice de diversidad para las estaciones de muestreo en cerro Mecana

\begin{tabular}{lcccc}
\hline Índices & \multicolumn{4}{c}{ Estaciones de muestreo } \\
& E1 & E2 & E3 & E4 \\
\hline Taxa_S & 73 & 61 & 52 & 38 \\
Individuals & 143 & 117 & 138 & 90 \\
Simpson_1-D & 0,9715 & 0,9608 & 0,9129 & 0,96 \\
Shannon_H & 3,962 & 3,711 & 3,162 & 3,35 \\
Margalef & 14,57 & 12,62 & 10,37 & 7,69 \\
Equitability_J & 0,9235 & 0,9028 & 0,8002 & 0,94 \\
\hline
\end{tabular}

y Myristicaceae), de las cuales Myristicaceae es la que tiene mayor cantidad de especies (2) en categorías de amenazas propiamente dicha. En ese sentido, una especie encuentra en riesgo extremadamente alto de extinción en estado silvestre en un futuro inmediato (Humiriastrum procerum), dos se enfrentan a un riesgo muy alto de extinción en estado silvestre en un futuro cercano(Aspidospermacruentum, Licania platypus), tres presentan alto riesgo de extinción en estado silvestre a medio plazo (Licania chocoensis, $\mathrm{Hu}$ - berodendron patinoi, y Otoba lehmannii). Otra especie se encuentra listada en el Apéndice II de la CITES (Cyathea delgadii), y las demás no satisfacen criterios de amenaza y se consideran en preocupación menor (Tabla 2).

Así entonces, una especie encuentra en riesgo extremadamente alto de extinción en estado silvestre en un futuro inmediato CR (Humiriastrum procerum ), dos se enfrentan a un riesgo muy alto de extinción en estado silvestre en un futuro cercano EN (Aspidosperma cruentum,

Tabla 2. Flora de interés especial y su estado de conservación

\begin{tabular}{lllc}
\hline Nombre vulgar & \multicolumn{1}{c}{ Especie } & Familia & Categoría \\
\hline Mil pesos & Oenocarpus bataua & & LC \\
Palma zancona & Socratea exorrhiza & & LC \\
Palma cuchilleja & Asterogyne martiana & & LC \\
Palma don Pedrito & Oenocarpus minor & Arecaceae & LC \\
NN & Phytelephas seemannii & & NT \\
Palma amargo & Welfia regia & & LC \\
Palma chascarrá & Bactris barronis & & LC \\
Palma tagua & Phytelephas seemannii & & NT \\
Costillo & Aspidosperma cruentum & Apocynaceae & EN \\
Carbonero & Licania chocoensis & Chrysobalanaceae & VU B1ab (iii)-Global \\
Tací & Cyathea delgadii & Cyatheaceae & CITES Apéndice II \\
Chanó & Humiriastrum procerum & Humiriaceae & CR A2acd \\
Carrá & Huberodendron patinoi & Malvaceae & VU A2cd-Nacional \\
Otobo & Otoba lehmannii & Myristicaceae & VU A4acd-Nacional \\
Incibe / tuave canelo & Licania platypus & & EN B1ab(iii)-Nacional \\
\hline
\end{tabular}

*NT: Casi amenazado; LC: Preocupación menor; VU: Vulnerable; EN: En peligro; CITES: Convention on International Trade in Endangered Species of Wild Fauna and Flora (Convención sobre el Comercio Internacional de Especies Amenazadas de Fauna y Flora Silvestres) 
Licania platypus), tres presentan alto riesgo de extinción en estado silvestre a medio plazo VU (Licania chocoensis, Huberodendron patinoi, y Otoba lehmannii); otra, aunque no satisface los criterios de Vulnerable, está próxima a hacerlo de forma inminente o en el futuro NT (Phytelephas seemannii); otra especie se encuentra listada en el Apéndice II de la CITES; mientras tanto, seis especies no cumplen ninguno de los criterios de las categorías UICN (Oenocarpus bataua, Socratea exorrhiza, Asterogyne martiana, Oenocarpus minor, Welfia regia, Bactris barronis) (Tabla 2).

\section{Consideraciones finales}

La variación de la composición y estructura florística medida que se asciende en el gradiente altitudinal del cerro Mecana expresa la existencia de particularidades relacionadas con el hábitat y comportamiento de algunas especies a diferentes rangos altitudinales, lo que sugiere que la exclusividad de algunas especies a determinadas zonas del gradiente, parece indicar que los patrones de distribución de la vegetación a lo largo del cerro Mecana, están relacionados con las particularidades del hábitat en cada estación.

Si bien es cierto que el gradiente altitudinal en el cerro mecana, ha sido objeto de algunas actividades antrópicas (IIAP 2014), también es cierto que estos impactos aún no se han hecho extensivos a todo el cerro, lo que permite mantener su diversidad florística e inclusive algunas especies consideradas en amenaza, dando lugar a la posibilidad de seguir comprendiendo las dinámicas intrínsecas de este ecosistema.

Por otra parte, aunque las especies identificadas con algún grado de amenaza no son numerosas con respecto a la riqueza de especies en el cerro, es de anotar que por el tipo de uso que estas presentan puede llegar a potenciarse sus condiciones de amenazas debido a la falta de control que las autoridades competentes hacen sobre este ecosistema, porque la tendencia de las poblaciones humanas es a ampliar cada vez más los espacios de uso a fin de satisfacer sus necesidades.

\section{Literatura citada}

Arruda DM, Brandão DO, Costa FV, Tolentino GS, Brasil RD, Neto SD, et al. 2011. Structural aspects and floristic similarity among tropical dry forest fragments with different management histories in northern Minas Gerais, Brazil. Rev Arvore. 35 (1): 131-42. Disponible en: http://www.scielo.br/scielo.php?script $=$ sci arttext\&pid=S0100-67622011000100016

AspillaA, Mosquera C, Valoyes H, Cuesta H, García F. 2003. Composición florística de un bosque pluvial Tropical (bp-T) en la parcela permanente de investigación en biodiversidad (PPIB) en Salero Unión Panamericana, Chocó. En: García F, Ramos Y, Palacios J, Arroyo J, Mena A, García M (eds.). Salero - Diversidad biológica de un bosque pluvial Tropical (bp-T); 39-44 pp.

Boyle BL. 1996. Changes on altitudinal and latitudinal gradients in neotropical montane forest. Saint Louis: Washington University; $550 \mathrm{pp}$.

Boyle B, Hopkins N, Lu Z, Raygoza JA, Mozzherin D, Rees T, et al. 2013. The taxonomic name resolution service: an online tool for automated standardization of plant names. BMC Bioinformatics. 14: 16. Disponible en: https://bmcbioinformatics.biomedcentral.com/ articles/10.1186/1471-2105-14-16

Brown J.H. 1995. Macroecology. Chicago: University of Chicago Press; 270 pp.

Brown JH, Lomolino MV. 1998. Biogeography. $2^{\text {nd }}$ ed. Sunderland: Sinauer Associates, Inc. Publishers.

Cardelús CL, Colwell RK, Watkins JEJr. 2006. Vascular epiphyte distribution patterns: explaining the mid-elevation richness peak. JEcol. 94 (1): 144-56. Disponible en: http://onlinelibrary.wiley.com/doi/10.1111/j.13652745.2005.01052.x/abstract

Colwell RK, Hurtt GC. 1994. Nonbiological gradients in species richness and a spurious rapoport effect. Am Nat. 144 (4): 570-95. Disponible en: https://www.jstor.org/ stable/2462939?seq=1\#page scan tab contents

Cortés A. 1981. Los suelos del andén Pacífico y su aptitud de uso. Bogotá: Instituto Geográfico «Agustín Codazzi». Disponible en: http://documentacion.ideam.gov.co/cgibin/koha/opac-detail.pl?biblionumber $=10989$ \&shelfbrowse itemnumber $=11550$

Cortés A. 1993. Los suelos. pp. 148-55. En: Leyva P (ed.). Colombia Pacífico. Tomo 1. Bogotá: Fondo FEN-Colombia.

Dobzhansky T. 1950. Evolution in the tropics. Am Sci. 38: 209-21. Disponible en: http://people.wku.edu/charles. smith/biogeog/DOBZ1950.htm

Gaston KJ, Williams PH, Eggleton P, Humphries CJ. 1995. Large-scale geographic patterns of biodiversity: Spatial 


\section{Bioetnia Volumen 14, 2017}

variation in family richness. Proc Royal Soc: Biol Sci. 260: $149-54$.

Gentry AH. 1993. A field guide to the families and genera of woody plants of northwest South America. Conservation International; 920 pp. Disponible en: http://press.uchicago.edu/ucp/books/book/chicago/F/bo5952254.html

Hernández-Stefanoni JL, Dupuy JM, Tun-Dzul F, May-Pat F. 2011. Influence of landscape structure and stand age on species density and biomass of a tropical dry forest across spatial scales. Landscape Ecol. 26 (3): 355-70. Disponible en: https://link.springer.com/article/10.1007/ $\underline{\text { s10980-010-9561-3 }}$

Holdridge LR. 1986. Ecología basada en zonas de vida. San José: Instituto Interamericano de Cooperación para la agricultura; $216 \mathrm{pp}$.

Instituto de Investigaciones Ambientales del Pacífico (IIAP), Corporación Autónoma Regional para el Desarrollo Sostenible del Chocó (Codechocó). 2011. Caracterización ecológica de ecosistemas aislados de la cordillera Occidental, cerro Galápagos, cerro de Tacarcuna y Alto del Buey. Informe Técnico. Quibdó: IIAP, Codechocó; 265 pp. Disponible en: https://siatpc.iiap.org.co/docs/ avances/ccerrosaislados.pdf

Instituto de Investigaciones Ambientales del Pacífico (IIAP). 2012. Identificación y caracterización ecológica de ecosistemas de media montaña, Cerro Mecana. Informe final. Quibdó: IIAP.

Instituto de Investigaciones Ambientales del Pacífico (IIAP). 2013. Caracterización ecológica y sociocultural del cerro Jánano, territorio sagrado para comunidades étnicas de la costa Pacífica chocoana, municipio de Nuquí, Chocó. Informe Técnico. Quibdó: IIAP; 117 pp. Disponible en: https://siatpc.iiap.org.co/docs/avances/ caracterizacion_ecologica_y_sociocultural_del_cerro janano territorio_sagrado_para_comunidades_etnicas de la costa pacifica chocoana.pdf

Instituto de Investigaciones Ambientales del Pacífico (IIAP). 2014. Evaluación de la integralidad ecosistémica y el estado de conservación del cerro Murrucucú, municipio de Tierra Alta-Córdoba. Chocó Biogeográfico equipo de trabajo. Informe final. Quibdó: IIAP; 44 pp.

Instituto de Investigaciones Ambientales del Pacífico (IIAP). 2015. Caracterización ecológica y sociocultural del cerro Mutis. Informe Técnico. Quibdó: IIAP; 125 pp.

Instituto de Investigaciones Ambientales del Pacífico (IIAP). 2016. Monitoreo del cerro Mecana. Informe final. Proyecto identificación y caracterización ecológica de ecosistemas de media montaña. Quibdó: IIAP.

Kappelle M, Zamora N. 1995. Change in woody species richness along an altitudinal gradient in Talamanca montane Quercus forest, Costa Rica. pp. 135-48. En: Churchill SP, Baslev H, Forero E, Luteyn JL (eds.). Biodiversity and conservation of Neotropical montane forest. New York: The New York Botanical Garden.

Kessler M. 2000. Elevational gradients in species richness and endemism of selected plant groups in the central Bolivian Andes.PlantEcol.149(2): 181-93. Disponible en:https:// link.springer.com/article/10.1023/A:1026500710274

Kessler M, Herzog SK, Fjeldså J, Bach K. 2001. Species richness and endemism of plant and bird communities along two gradients of elevation, humidity and land use in the Bolivia Andes. Diversity and Distributions. 7(1-2): 61-77. Disponible en: http://onlinelibrary.wiley. com/doi/10.1046/j.1472-4642.2001.00097.x/abstract

Kessler, M. 2002. The elevational gradient of Andean plant endemism: varying influences of taxon-specific traits and topography at different taxonomic levels. JBiogeogr. 29 (9): 1159-65. Disponible en: http://onlinelibrary.wiley. com/doi/10.1046/j.1365-2699.2002.00773.x/abstract

Kessler M, Kluge J, Hemp A, Ohlemüller R. 2011. A global comparative analysis of elevational species richness patterns of ferns. Global Ecol Biogeograp. 20 (6): 868-80. Disponible en: http://onlinelibrary.wiley.com/ doi/10.1111/j.1466-8238.2011.00653.x/abstract

Kitayama K. 1992. An altitudinal transect study of the vegetation of Mount Kinabalu, Borneo. Vegetatio.102 (2): 149-71. Disponible en: https://link.springer.com/ article/10.1007/BF00044731

Kitayama K, Mueller-Dombois D. 1994. An altitudinal transect analysis of the windward vegetation on Haleakala, a Hawaiian island mountain: (2) vegetation zonation. Phytocoenol Band. 24 (1-4): 135-54. Disponible en: https:// www.schweizerbart.de/papers/phyto/detail/24/81002/ An altitudinal transect analysis of the windward vegetation_on_Haleakala_a_Hawaiian_island_mountain 2 vegetation zonation

Koleff P, Gaston KJ. 2001. Latitudinal gradients in diversity: real patterns and random models. Ecography. 24 (3): 341-51. Disponible en: http://onlinelibrary.wiley.com/ doi/10.1111/j.1600-0587.2001.tb00207.x/abstract

Krömer T, Kessler M, Gradstein SR, Acebey A. 2005. Diversity patterns of vascular epiphytes along an elevational gradient in the Andes. J Biogeograp, 32 (10): 1799-809. Disponible en: https://www.jstor.org/stable/3566350?se$\mathrm{q}=1$ \#page scan tab contents

Lamprecht H. 1990. Silvicultura en los trópicos/Los ecosistemas forestales en los bosques tropicales y sus especies arbóreas -posibilidades y métodos para un aprovechamiento sostenido. Deutsche Gesellschaft für Technische Zusammenarbeit (GTZ) GmbH. Eschborn.

Leiva JA. 2001. Comparación de las estrategias de regeneración natural entre los nosques primarios y secundarios en las zonas bajas del Atlántico costarricense. Tesis Bach. Cartago: Escuela de Ingeniería Foretal, Instituto Tecnológico de Costa Rica. 112pp. Disponible en: $\underline{\text { https:// }}$ repositoriotec.tec.ac.cr/bitstream/handle/2238/5656/ comparacion $\% 20$ estrategias $\% 20$ regeneracion $\% 20$ natural $\% 20$ bosques $\% 20$ atlantico.pdf?sequence $=1 \&$ isAllowed $=\mathrm{y}$

Lieberman D, Lieberman M, Peralta R, Harsthorn GS. 
1996. Tropical forest structure and composition on a large-scale altitudinal gradient in Costa Rica. $J$ Ecol. 84 (2): 137-52. Disponible en: https://www.jstor.org/ stable/2261350?seq=1\#page scan tab contents

Lomolino MV.2001. Elevational gradients of species-density: historical and prospective views. Global Ecol Biogeograp. 10 (1): 3-13. Disponible en: http://onlinelibrary. wiley.com/doi/10.1046/j.1466-822x.2001.00229.x/ abstract

MacArthur RH. 1969. Patterns of communities in the tropics. Biol J Lin Soc. 1 (1-2): 19-30. Disponible en: http:// onlinelibrary.wiley.com/doi/10.1111/j.1095-8312.1969. tb01809.x/abstract

MacArthur H. 1972. Geographical ecology. Patterns in the distribution of species. Princeton: Princeton University Press; 269 pp. Disponible en: https://www.amazon. com/Geographical-Ecology-Robert-H-MacArthur/ $\mathrm{dp} / 0691023824$

McCain CM, Grytnes J-A. 2010. Elevational gradients in species richness. In: Encyclopedia of Life Sciences (ELS). Chichester: John Wiley \& Sons, Ltd. Disponible en: https://onlinelibrary.wiley.com/doi/ pdf/10.1002/9780470015902.a0022548

Mendoza H, Ramírez B. 2006. Guía ilustrada de géneros de Melastomataceae y Memecylaceae de Colombia. Bogotá: Instituto Alexander von Humboldt; 300 pp.

Mendoza H, Ramírez B, Jiménez LC. 2004. Rubiaceae de Colombia. Guía ilustrada de géneros. Bogotá: Instituto Alexander von Humboldt; 321 pp.

Mendoza-Cifuentes H. 2012. Patrones de riqueza específica de las familias Melastomataceae y Rubiaceae en la cordillera oriental de Colombia, norte de los Andes y consideraciones para la conservación. Rev. Colomb Forestal. 15 (1): 5-54. Disponible en: https://revistas.udistrital. edu.co/ojs/index.php/colfor/article/view/3711/5310

Ministerio de Ambiente y Desarrollo Sostenibla. 2014. Resolución 0192 por la cual se establece el listado de especies silvestres amenazadas de Colombia. Bogotá: MADS.

Mittelbach GG, Schemske DW, Cornell HV, AllenAP, Brown JM, Bush MB, et al. 2007. Evolution and the latitudinal diversity gradient: speciation, extinction and biogeography. Ecol Letters. 10 (4): 315-31. Disponible en: https://onlinelibrary.wiley.com/doi/abs/10.1111/j.14610248.2007.01020.x

Mosquera LJ, Robledo D, Asprilla A. 2007. Diversidad florística de dos zonas de bosque tropical húmedo en el municipio de Alto Baudó, Chocó, Colombia. Acta Biol Colomb. 12: 75-90. Disponible en: https://revistas.unal. edu.co/index.php/actabiol/article/view/27244

Nathan JS. 2002. Elevational gradients in ant species richness: area, geometry, and Rapaport's rule. Ecogeography. 25: 25-32. Disponible en: https://onlinelibrary.wiley.com/ doi/abs/10.1034/j.1600-0587.2002.250104.x

Oliveira YMM de, Rotta E. 1982. Levantamento horizontal de uma mata de araucaria do primeiro planalto paranaense.
Bol Pesquisa. 4: 1-39.

Pardo P, Cediel J. 1994. Composición y diversidad florística de los bosques de cabo Corrientes, costa pacifica del Chocó. En: Memorias del I Congreso Nacional Sobre Biodiversidad. Biopacífico. Cali diciembre 4 al 7 de 1994. Universidad del Valle, Instituto de Estudio del Pacífico; pp. 85-92.

Rahbek C. 1995. The elevational gradient of species richness: a uniform pattern? Ecography. 18: 200-5. Disponible en: https://onlinelibrary.wiley.com/doi/ abs/10.1111/j.1600-0587.1995.tb00341.x

Ricklefs RE. 2003. A comprehensive framework for global patterns in biodiversity. Ecol Letters 7: 1-15. Disponible en: https://onlinelibrary.wiley.com/doi/pdf/10.1046/ j.1461-0248.2003.00554.X

Rodríguez C, Brenes L. 2009. Estructura y composición florística de dos remanentes de bosque premontano muy húmedo en la reserva madre verde palmares Costa Rica. Rev Pensamiento Actual. 9 (12-13): 115-24. Disponible en: https://revistas.ucr.ac.cr/index.php/pensamiento-actual/article/view/2841

Rosenzweig ML. 1995. Species diversity in space and time. Tucson: The University of Arizona.

Sanders NJ, Rahbek C. 2012. The patterns and causes of elevational diversity gradients. Ecography. 35: 1-3. Disponible en: https://onlinelibrary.wiley.com/doi/ abs/10.1111/j.1600-0587.2011.07338.x

Schoener TW. 1976. The species-area relation within archipelagoes: models and evidence from island land birds. In: Firth HJ, Calaby JH (eds). Proceedings of the 16th International Ornithological Congress. Canberra, Australia, 12-17 August 1974.

Stevens GC. 1989. The latitudinal gradient in geographical range: how so many species coexist in the tropics. $\mathrm{Am}$ Nat. 133 (2): 240-56. Disponible en: https://www.jstor. org/stable/2462300?seq=1\#page scan tab contents

Stevens GC. 1992. The elevational gradient in altitudinal range: an extension of Rapoport's latitudinal rule to altitude. Am Nat. 140 (6): 893-911. Disponible en: https:// www.ncbi.nlm.nih.gov/pubmed/19426029

Vázquez JA, Givnish TJ. 1998. Altitudinal gradients in tropical forest composition, structure, and diversity in the Sierra de Manantlán. J Ecol. 86: 99-1020.

Villarreal H, Álvarez M, Córdoba S, Escobar F, Fagua G, Gast F, et al. 2006. Manual de métodos para el desarrollo de inventarios de biodiversidad. $2^{\mathrm{a}}$ ed. Programa de inventarios de biodiversidad. Bogotá: Instituto de Investigación de Recursos Biológicos Alexander von Humboldt; 236 pp.

Yockteng R, Cavelier J. 1998. Diversidad y mecanismos de dispersión de árboles de la Isla Gorgona y de los bosques húmedos tropicales del Pacífico colombo-ecuatoriano. Rev Biol Trop. 46 (1): 45-53. Disponible en: https://revistas.ucr.ac.cr/index.php/rbt/article/view/19352/19401 


\section{Bioetnia Volumen 14, 2017}

Anexo

Composición florística en el cerro Mecana

\begin{tabular}{|c|c|c|c|c|c|c|}
\hline Nombre vulgar & Familia & Nombre científico & E1 & E2 & E3 & E4 \\
\hline NN & Euphorbiaceae & Alchornea coelophylla Pax \& K. Hoffm. & $x$ & & & \\
\hline Taparo & Euphorbiaceae & Alchornea grandiflora Müll. Arg. & $x$ & & $\mathrm{x}$ & \\
\hline Palmillo & Fabaceae & Andira surinamensis (Bondt) Splitg. ex Pulle & $x$ & $x$ & $\mathrm{x}$ & \\
\hline Jigua negro & Lauraceae & Aniba puchury-minor (Mart.) Mez & $x$ & $x$ & & \\
\hline NN & Araceae & Anthurium hacumense Engl. & & & $\mathrm{x}$ & \\
\hline NN & Araceae & Anthurium lancifolium Schott & & & $\mathrm{x}$ & \\
\hline NN & Araceae & Anthurium myosuroides (Kunth) Schott & & & & $x$ \\
\hline NN & Araceae & Anthurium trilobum hort. ex André & $x$ & $x$ & & $x$ \\
\hline NN & Acanthaceae & Aphelandra lingua-bovis Leonard. & $x$ & $x$ & $x$ & \\
\hline Costillo & Apocynaceae & Aspidosperma cruentum Woodson & $x$ & $x$ & & \\
\hline Costillo acanalado & Apocynaceae & Aspidosperma desmanthum Benth. ex Müll. Arg. & $x$ & & $x$ & $x$ \\
\hline Palma cuchilleja & Arecaceae & Asterogyne martiana (H. Wendl.) H. Wendl. ex Hemsl. & $x$ & $x$ & $x$ & \\
\hline Wuerregue & Arecaceae & Astrocaryum standleyanum L.H. Bailey & $\mathrm{x}$ & & & \\
\hline Palma chascará & Arecaceae & Bactris barronis L.H. Bailey & $x$ & $\mathrm{x}$ & & \\
\hline NN & Arecaceae & Bactris sp & $x$ & & & \\
\hline NN & Melastomataceae & Blakea podagrica Triana. & $x$ & & & \\
\hline Sande & Moraceae & Brosimum utile (Kunth) Oken & $x$ & & $x$ & \\
\hline Clavellino & Fabaceae & Brownea rosa-de-monte P.J. Bergius & & & & $x$ \\
\hline Caraño & Burseraceae & Bursera tomentosa (Jacq.) Triana \& Planch. & & & $\mathrm{x}$ & \\
\hline Aceite & Clusiaceae & Calophyllum mariae Planch. \& Triana & $x$ & $x$ & $x$ & \\
\hline NN & Arecaceae & Calyptrogyne ghiesbreghtiana (Linden \& H. Wendl.) H. Wendl. & $x$ & & & \\
\hline Wina & Meliaceae & Carapa guianensis Aubl. & $x$ & $x$ & $x$ & $x$ \\
\hline NN & Achariaceae & Carpotroche sp & & & & $x$ \\
\hline NN & Ericaceae & Cavendishia bracteata (Ruiz \& Pav. ex J. St.-Hil.) Hoerold & $x$ & & & \\
\hline NN & Ericaceae & Cavendishia complectens Hemsl. & & $x$ & & \\
\hline Zanca de araña & Clusiaceae & Chrysochlamys floribunda Cuatrec. & $x$ & $x$ & $x$ & $x$ \\
\hline Caimito piedra & Sapotaceae & Chrysophyllum argenteum Jacq. & $x$ & $x$ & $x$ & \\
\hline NN & Sapotaceae & Chrysophyllum cainito L. & & & $x$ & \\
\hline NN & Ciclantaceae & Cyclanthus bipartitus Poit. ex A. Rich. & $x$ & $x$ & $x$ & \\
\hline NN & Melastomataceae & Clidemia aff hirta & & & $x$ & \\
\hline NN & Meliaceae & Cordia sp & & & $x$ & \\
\hline NN & Costaceae & Costus scaber Ruiz \& Pav. & & & & $x$ \\
\hline NN & Rubiaceae & Coussarea sp & & & & $\mathrm{x}$ \\
\hline NN & Euphorbiaceae & Croton brevipes Pax. & $x$ & $x$ & & \\
\hline NN & Sapotaceae & Chrysophyllum oliviforme L. & & & $\mathrm{x}$ & \\
\hline Tací & Cyatheaceae & Cyathea delgadii & $x$ & $x$ & & \\
\hline NN & Cyatheaceae & Cyathea sp & & & $x$ & \\
\hline NN & Ciclantaceae & Cylanthus bipartitus Poit. ex A. Rich. & $x$ & & & \\
\hline NN & Malvaceae & Dendropanax arboreus (L.) Decne. \& Planch. & $x$ & $x$ & & \\
\hline NN & Araliaceae & Dendropanax sp & $x$ & & & \\
\hline Matamba & Arecaceae & Desmoncus cirrhifer A.H. Gentry \& Zardini & $x$ & $x$ & & \\
\hline Sangre gallo & Myristicaceae & Dialyanthera acuminata Standl. & & & & $x$ \\
\hline
\end{tabular}


Análisis florístico altitudinal. R Rengifo-Ibargüen

Anexo

Composición florística en el cerro Mecana (continuación)

\begin{tabular}{|c|c|c|c|c|c|c|}
\hline Nombre vulgar & Familia & Nombre científico & E1 & E2 & E3 & E4 \\
\hline NN & Commelinaceae & Dichorisandra hexandra (Aubl.) C.B. Clarke & $x$ & $\mathrm{x}$ & & \\
\hline Bagata & Fabaceae & Dussia lehmannii Harms & & $x$ & & \\
\hline NN & Lecythidaceae & Eschweilera amplexifolia S.A. Mori & & & $x$ & $\mathrm{x}$ \\
\hline Guasca & Lecythidaceae & Eschweilera panamensis Pittier & & & & $x$ \\
\hline NN & Lecythidaceae & Eschweilera pittieri R. Knuth & & $x$ & & \\
\hline NN & Arecaceae & Euterpe $s p$ & & & $x$ & \\
\hline Caimitillo & Rubiaceae & Faramea calophylla Standl. & $x$ & $x$ & $\mathrm{x}$ & \\
\hline NN & Clusiaceae & Garcinia madruno (Kunth) Hammel & $x$ & & & \\
\hline NN & Gesneriaceae & Gasteranthus delphinioides (Seem.) Wiehler. & & & & $\mathrm{x}$ \\
\hline NN & Arecaceae & Geonoma cuneata H. Wendl. ex Spruce & $x$ & $x$ & $x$ & \\
\hline NN & Arecaceae & Geonoma deversa (Poit.) Kunth & & $x$ & $x$ & \\
\hline NN & Arecaceae & Geonoma stricta (Poit.) Kunth & & & $\mathrm{x}$ & \\
\hline Carbonero & Annonaceae & Guatteria cargadero Triana \& Planch. & & $x$ & & \\
\hline NN & Rubiaceae & Guettarda crispiflora & & $x$ & & \\
\hline NN & Gesneriaceae & Guzmania glomerata Mez \& Wercklé & $x$ & & & \\
\hline NN & Bromeliaceae & Guzmania sp2 & $x$ & & & \\
\hline NN & Bromeliaceae & Guzmania musaica (Linden \& André) Mez & $x$ & & $x$ & $x$ \\
\hline NN & Bromeliaceae & Guzmania sp & $x$ & & & $x$ \\
\hline NN & Rubiaceae & Hippotis albiflora H. Karst. & $x$ & $x$ & & \\
\hline Carrá & Malvaceae & Huberodendron patinoi Cuatrec. & & $x$ & & \\
\hline Chanó & Humiriaceae & Humiriastrum procerum (Little) Cuatrec. & $x$ & $x$ & $x$ & $x$ \\
\hline NN & Arecaceae & Hyospathe elegans Mart. & $x$ & & $x$ & \\
\hline Guamo cajeto & Fabaceae & Inga coruscans Humb. \& Bonpl. ex Willd. & & & & $x$ \\
\hline Guamo de loma & Fabaceae & Inga sp & $x$ & & & \\
\hline Aceite & Fabaceae & Inga vera Willd. & $x$ & & & $\mathrm{x}$ \\
\hline Sangre gallina & Myristicaceae & Iryanthera hostmannii (Benth.) Warb. & $x$ & $x$ & & $x$ \\
\hline NN & Marantaceae & Ischnosiphon arouma (Aubl.) Körn. & $x$ & $x$ & & \\
\hline NN & Apocynaceae & Lacmellea sp & & $x$ & & \\
\hline Carbonero & Chrysobalanaceae & Licania chocoensis Cuatrec. & & & $x$ & \\
\hline Incibe/tuave canelo & Myristicaceae & Licania platypus (Hemsl.) Fritsch & & & $x$ & \\
\hline Carbonero & Chrysobalanaceae & Licania macrocarpa Cuatrec. & $x$ & & & $\mathrm{x}$ \\
\hline Tuave & Lauraceae & Licaria triandra (Sw.) Kosterm. & $x$ & & $\mathrm{x}$ & \\
\hline Garrapato & Fabaceae & Lonchocarpus sericeus (Poir.) Kunth ex DC. & & & $x$ & \\
\hline NN & Euphorbiaceae & Mabea chocoensis Cutrec. & $x$ & $x$ & & \\
\hline Ají & Apocynaceae & Mandevilla hirsuta (Rich.) K. Schum & $x$ & $x$ & & \\
\hline Oquendo & Moraceae & Maquira $s p$ & $x$ & $x$ & & $x$ \\
\hline NN & Malvaceae & Matisia castano H. Karst. \& Triana & $x$ & $x$ & $x$ & \\
\hline Almirajocillo & Malvaceae & Matisia sp1 & $x$ & $x$ & $x$ & \\
\hline Hormigo colorado & Melastomataceae & Miconia nervosa (Sm.) Triana & $x$ & $x$ & $x$ & $x$ \\
\hline Palma gua guarín & Melastomataceae & Miconia punctata (Desr.) D. Don ex DC. & & & $x$ & \\
\hline NN & Melastomataceae & Miconia sp & $x$ & $x$ & & \\
\hline NN & Melastomataceae & Mouriri sp & & & $x$ & $x$ \\
\hline Jigua negro & Lauraceae & Ocotea cernua (Nees) Mez. & $x$ & & $x$ & \\
\hline
\end{tabular}




\section{Bioetnia Volumen 14, 2017}

Anexo

Composición florística en el cerro Mecana (continuación)

\begin{tabular}{|c|c|c|c|c|c|c|}
\hline Nombre vulgar & Familia & Nombre científico & E1 & E2 & E3 & E4 \\
\hline Raspadillo & Lauraceae & Ocotea $s p$ & $x$ & & $x$ & \\
\hline Mil pesos & Arecaceae & Oenocarpus bataua Mart. & $x$ & $x$ & & \\
\hline Palma don Pedrito & Arecaceae & Oenocarpus minor Mart. & $x$ & $x$ & & \\
\hline Otobo & Myristicaceae & Otoba lehmannii (A.C. Sm.) A.H. Gentry & & & $x$ & \\
\hline NN & Fabaceae & Pentaclethra macroloba (Willd.) Kuntze & & $x$ & & \\
\hline Cauchillo de loma & Moraceae & Perebea guianensis Aubl. & $x$ & $x$ & & $\mathrm{x}$ \\
\hline NN & Moraceae & Perebea xanthochyma H. Karst. & $x$ & $\mathrm{x}$ & & \\
\hline NN & Araceae & Philodendron fenzlii Engl. & & & $x$ & \\
\hline Palma tagua & Arecaceae & Phytelephas seemannii O.F. Cook & $x$ & $\mathrm{x}$ & & \\
\hline Caimito & Sapotaceae & Pouteria cuspidata (A. DC.) Baehni & $\mathrm{x}$ & $\mathrm{x}$ & $\mathrm{x}$ & $\mathrm{x}$ \\
\hline NN & Sapotaceae & Pouteria multiflora (A. DC.) Eyma & $x$ & & & $x$ \\
\hline Caimito popa & Lauraceae & Pouteria sp & $x$ & & & $x$ \\
\hline NN & Arecaceae & Prestodea decurrens (H. Wendl. ex Burret) H.E. Moore & $x$ & $\mathrm{x}$ & & \\
\hline Anime & Burceraceae & Protium nervosum Cuatrec. & & & $x$ & $x$ \\
\hline NN & Rubiaceae & Psychotria capitata Ruiz \& Pav. & & & $x$ & \\
\hline NN & Rubiaceae & Psychotria cf marginata & $\mathrm{x}$ & $\mathrm{x}$ & & $x$ \\
\hline NN & Rubiaceae & Psychotria cincta Standl. & $x$ & $x$ & $x$ & $x$ \\
\hline NN & Rubiaceae & Psychotria elata (Sw.) Hammel & & $x$ & & $x$ \\
\hline NN & Rubiaceae & Psychotria pilosa Ruiz \& Pav. & & $x$ & & \\
\hline NN & Malvaceae & Quararibea bracteolosa (Ducke) Cuatrec. & $x$ & & & $x$ \\
\hline NN & Araceae & Rhodospatha cf moritziana & & $x$ & & \\
\hline Cucharo & Simaroubaceae & Simaruba $s p$ & $x$ & & $x$ & $\mathrm{x}$ \\
\hline Palma zancona & Arecaceae & Socratea exorrhiza (Mart.) H. Wendl. & $x$ & $x$ & $x$ & \\
\hline NN & Apocynaceae & Aspidosperma cruentum Woodson & & $x$ & & \\
\hline NN & & Syngonium sp & & & $x$ & \\
\hline NN & Araceae & Syngonium triphyllum Birdsey ex Croat & & & $x$ & \\
\hline NN & Helechos & Tectaria sp & $x$ & $x$ & $x$ & $x$ \\
\hline $\mathrm{NN}$ & Clusiaceae & Tovomita nicaraguensis (Oerst., Planch. \& Triana) L.O. Williams & & $x$ & & \\
\hline Zanca de araña & Clusiaceae & Tovomita weddelliana Planch. \& Triana & $x$ & $x$ & & \\
\hline Machare & Hypericaceae & Vismia macrophylla Kunth & $x$ & $x$ & & $x$ \\
\hline Palo santo & Vochysiaceae & Vochysia ferruginea Stafleu & $x$ & $\mathrm{x}$ & & $\mathrm{x}$ \\
\hline Palma & Arecaceae & Welfia georgii $\mathrm{H}$. Wendl. & & $x$ & & \\
\hline Palma amargo & Arecaceae & Welfia regia Mast. & $x$ & $x$ & $x$ & \\
\hline Palma memé & Arecaceae & Wettinia quinaria (O.F. Cook \& Doyle) Burret. & $x$ & $\mathrm{x}$ & $x$ & \\
\hline NN & Arecaceae & Iriartea deltoidea Ruiz \& Pav. & $x$ & & & \\
\hline Total & & & 75 & 61 & 52 & 38 \\
\hline
\end{tabular}

